\title{
Особенности измерения удельного и поверхностного сопротивлений четырехзондовым методом
}

И. Васильев ${ }^{1}$

УДК 658.5:621.317| ВАК 05.27.06

\begin{abstract}
При производстве изделий микроэлектроники качество материалов играет критическую роль. Одним из способов оценки качества пластин или созданных функциональных слоев является измерение удельного и поверхностного сопротивлений четырехзондовым методом. Эти электрофизические параметры позволяют осуществить входной контроль материалов, выявить недостатки технологического процесса, а также спрогнозировать процент разброса характеристик конечных изделий. В данной статье приведен обзор современных методов измерения удельного и поверхностного сопротивлений, описаны преимущества и недостатки каждого из них, а также возможные способы устранения этих недостатков. В качестве готового решения представлены ручные и автоматические программно-аппаратные комплексы, которые способны решать различные задачи производства при измерении электрофизических параметров.
\end{abstract}

\section{ОСНОВНЫЕ ОПРЕДЕЛЕНИЯ}

Удельное электрическое сопротивление является фундаментальным параметром, который определяет способность материала препятствовать протеканию через него электрического тока. В отличие от широко известного электрического сопротивления, которое зависит от формы и площади поперечного сечения, удельное сопротивление не зависит от геометрических размеров, а характеризует исключительно электропроводящие свойства материала.

Ниже приведен закон Ома в классическом и дифференциальном видах:

$$
\begin{aligned}
& I=\frac{U}{R}, \\
& \vec{j}=\frac{\vec{E}}{\rho},
\end{aligned}
$$

где I - сила тока; U - напряжение; $\mathrm{R}$ - электрическое сопротивление; $\vec{j}$ - вектор плотности тока; $\vec{E}$ - вектор напряженности электрического поля; $\rho$ - удельное электрическое сопротивление.

Вторая формула применима для бесконечно малого объема, а потому наиболее удобна, когда мы исследуем новые материалы (в том числе анизотропные), такие как

ООО "Остек-Электро", руководитель направления

микроэлектроники, vasilyev.@@stec-group.ru. графен, углеродные нанотрубки и т.д. Как видно, единственным параметром, который отвечает за свойства самого материала, здесь является удельное электрическое сопротивление. В случае работы с тонкими слоями в полупроводниковом производстве также вводится понятие поверхностного сопротивления, связь которого с удельным сопротивлением рассмотрена ниже.

Электрическое сопротивление однородного образца, представленного на рис. 1, определяется следующим образом:

$$
\mathrm{R}=\frac{\rho l}{\mathrm{~S}}=\frac{\rho l}{w d}
$$

где $\mathrm{R}$ - электрическое сопротивление; $S$ - площадь поперечного сечения; $d$ - толщина материала; $w$ - ширина; $l$ - длина.

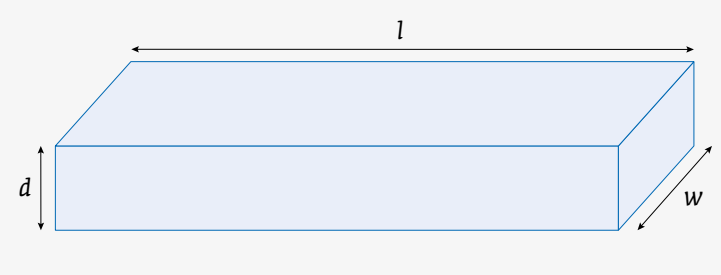

Рис. 1. Образец с линейными размерами $d, l$ и $w$ 
Если мы возьмем квадрат поверхности материала, то есть $l=w$, то из выражения (3) получим соотношение:

$$
\mathrm{R}_{\square}=\frac{\rho}{d}
$$

где $R_{\square}$ - поверхностное сопротивление [Ом/ ] (Ом на квадрат). Другими словами, поверхностное сопротивление представляет собой сопротивление квадратного участка поверхности материала толщиной d. Причем оно не зависит от величины сторон этого квадрата. Понятие поверхностного сопротивления также применимо и для неоднородно легированных слоев. С помощью данного параметра можно определить исходное качество материала, выявить проблемы технологического процесса при проведении межоперационного контроля отдельных слоев, а также осуществлять выходной контроль качества материала.

\section{МЕТОДЫ ИЗМЕРЕНИЯ}

На сегодняшний день существуют два основных метода измерения поверхностного сопротивления:

- четырехзондовый метод Кельвина;

- бесконтактный вихретоковый метод.

Вихретоковый метод предполагает взаимодействие образца с электромагнитным полем, которое формируется генератором (как правило, это катушка индуктивности). Возбуждаемые в образце вихревые токи в свою очередь создают электромагнитное поле, которое действует на катушку индуктивности, изменяя ее полное электрическое сопротивление (рис. 2). Таким образом можно определить поверхностное сопротивление образцов. Преимуществом данного метода являются отсутствие контакта с исследуемым образцом, высокая пропускная способность и высокое разрешение. В качестве недостатков можно отметить невысокую точность измерений (погрешность 10\%) и малый диапазон измерения сопротивления данный метод преимущественно используется для проводящих образцов. Поэтому оборудование, построенное

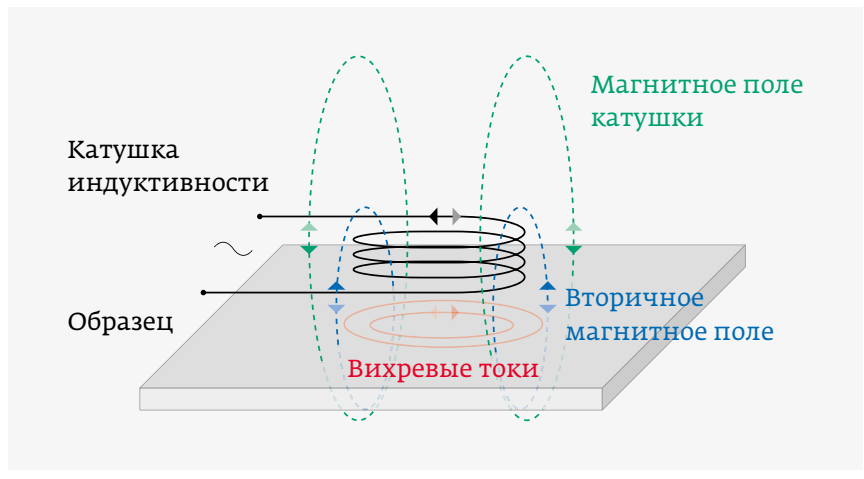

Рис. 2. Схематичное изображение принципа работы вихретокового метода на данном принципе, применяется в основном для in-line контроля при больших объемах производства.

Четырехзондовый метод Кельвина предполагает использование специальной измерительной головы с четырьмя иглами (рис. 3). Через крайние иглы (7 и 4) течет измерительный ток, через иглы 2 и 3 выполняется измерение напряжения с образца. Все иглы расположены на одинаковом расстоянии друг от друга. Данный метод позволяет значительно расширить диапазон измерения в область малых значений сопротивления за счет использования четырехпроводной схемы подключения и отсутствия падения напряжения на измерительных кабелях. Кроме того, он также может применяться для диэлектрических материалов с высоким значением сопротивления ( МОм). Точность измерений данным методом может быть лучше $\pm 1 \%$, а воспроизводимость составляет $\pm 0,1 \%$.

К основным недостаткам данного метода относятся:

1) наличие непосредственного контакта с образцом: иглы измерительной головы могут оставлять царапины или проколы измеряемого слоя;

2) нагрев образца вследствие протекания измерительного тока;

3) изменение расстояния между иглами измерительной головы вследствие ее износа;

4) термо-ЭдС из-за неидеальности контактов и неоднородности образца.

Эти недостатки могут быть устранены с помощью некоторых методик, которые мы рассмотрим отдельно более подробно.

1. Повреждение образца можно минимизировать путем подбора механических параметров измерительной головы, таких как радиус закругления и усилие прижатия игл. К примеру, при измерении параметров кремниевых пластин оптимальным вариантом будет использование диаметра закругления игл 40 мкм и усилия прижатия 200 г. Это связано с необходимостью создания надежного электрического контакта при наличии естественного

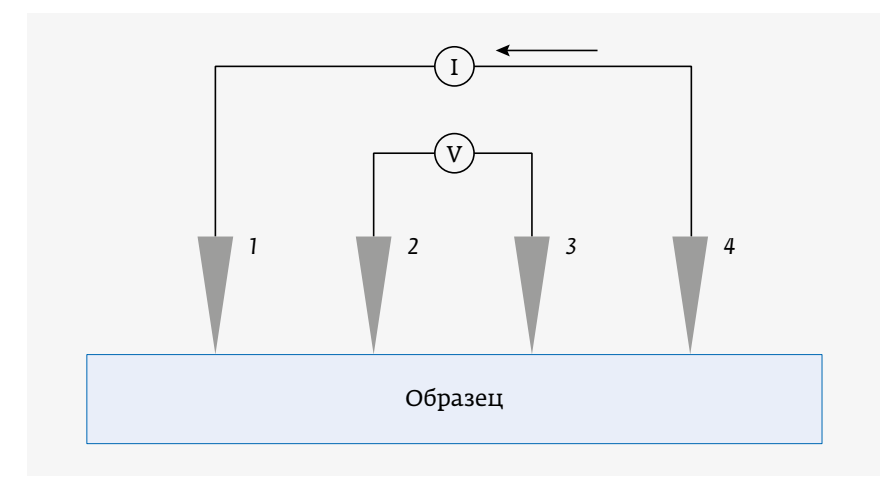

Рис. 3. Расположение игл на образце в четырехзондовом методе 
слоя окисла. В случае проведения тестирования более мягких материалов, например ITO, желательно использовать иглы с большим радиусом закругления (500 мкм) и меньшим усилием прижатия (25г).

2. Для уменьшения нагрева образца рекомендуется использовать импульсный режим измерения и такой уровень тока, который не позволит существенно разогреть образец за время измерения. На практике выбор величины тока обусловлен чувствительностью измерителя напряжения либо точностью источника тока. Как правило, большинство измерителей способно точно регистрировать сигналы порядка милливольт. Поэтому для материалов, поверхностное сопротивление которых лежит в диапазоне от единиц Ом / г до сотен кОм / , существует эмпирическое правило устанавливать измерительный ток такой величины, которая создаст падение напряжения на внутренних иглах от 7 до 15 мВ. Однако в случае проводящих материалов (мОм/ и менее) достичь указанного падения напряжения можно только при использовании довольно большого тока, что провоцирует нагрев образца. При измерении же высокорезистивных материалов (МОм/ $и$ и более) напряжение в несколько милливольт требует протекания тока величиной порядка наноампера, который может быть искажен внешними электромагнитными наводками. Оба пограничных варианта решаются по-разному в зависимости от тестируемого материала. Однако обобщенное правило для любого случая - это выбирать ток, который одновременно обеспечит наибольшее падение напряжения между иглами и не создаст значительного разогрева образца.

3. Для тонкого образца формула для расчета поверхностного сопротивления в общем случае выглядит следующим образом:

$$
\mathrm{R}_{\square}=\frac{\pi}{\ln 2} \frac{\mathrm{V}_{23}}{\mathrm{I}_{14}},
$$

где $V_{23}$ - напряжение между иглами 2 и 3; $I_{14}$ - измерительныЙ ток.

В данной формуле нет параметра, отвечающего за расстояние между иглами, - он сокращается, если этот параметр одинаков для всех игл. Поэтому если в ходе эксплуатации измерительной головы расстояние между иглами со временем изменяется, то это значительно влияет на результат измерения. Более того, ни один изготовитель измерительных голов не может обеспечить одинаковое расстояние между иглами с учетом того, что они являются подпружиненными, из-за чего фактическое расстояние в момент контактирования может меняться. В этом случае, согласно ГОСТ 24392-80 и ASTM F84-99, необходимо провести замер реального расстояния между иглами в момент контакта. Для этого осуществляется серия контактов с образцом и проводятся замеры фактического расстояния между иглами по следам игл на поверхности образца. Полученная информация позволяет рассчитать

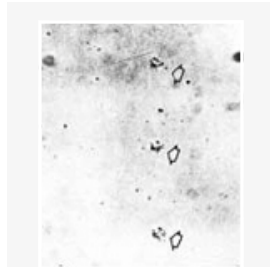

a)

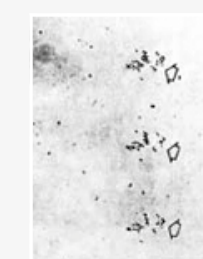

б)
Рис. 4. Фотографии следов от игл измерительной головы после контактирования с образцом: а - следы от острых игл; 6 - следы от затупленных игл; в - следы от игл при наличии латерального сдвига во время контактирования

эффективное значение межзондового расстояния и увеличить точность измерения. Такого рода измерения необходимо проводить время от времени, чтобы понимать текущее состояние измерительной головы. Более того, сама система перемещения головы должна обеспечивать строго перпендикулярное расположение игл на образце, исключая латеральное перемещение по образцу и его царапание (рис. 4в).

Для получения более достоверных результатов при измерении распределения поверхностного сопротивления по поверхности пластины часто прибегают к использованию одной из разновидностей четырехзондового метода метода самокомпенсации геометрическихэффектов (ASTM F1529). Этот метод имеет следующие преимущества:

- снижение влияния краевых эффектов до 0,1\% по сравнению с измерениями в центре;

- не требуется информация о диаметре образца и точных координатах размещения измерительной головы на образце: поправочный коэффициент непосредственно рассчитывается с помощью двух схем измерения, представленных на рис. 5;

- процедуру измерения расстояния между иглами можно исключить, так как отклонения в расположении игл некритичны, как в традиционном методе измерения.

Таким образом можно нивелировать негативное влияние износа измерительной головы.

4. Как известно, термо-ЭДС $\left(V_{\text {темғ }}\right)$ возникает при контакте двух разнородных материалов, которые имеют разную температуру. Данное явление часто наблюдается при контакте измерительной головы и исследуемого образца. Более того, сам измеритель напряжения может иметь некоторое смещение относительно нуля $\left(V_{\text {of }}\right)$. Оба этих эффекта приводят к появлению ошибки при измерениях. Чтобы их исключить, в каждой точке на образце проводят два измерения с противоположными направлениями тока: сначала измеряют сопротивления при протекании тока от первой иглы к четвертой, а затем - от четвертой к первой. 


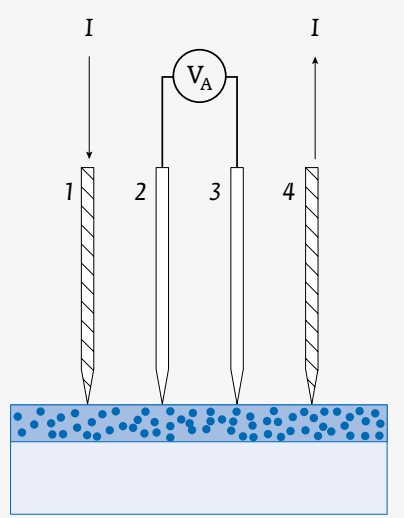

a)

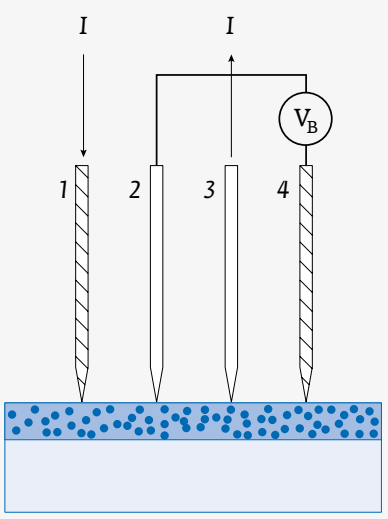

б)

Рис. 5. Схемы измерения: а - традиционная; 6 - дополнительная для компенсации геометрических эффектов

Полученные два значения поверхностного сопротивления используются для нахождения среднего значения, которое исключает термо-ЭДС и смещение измерителя напряжения, поскольку обе эти величины не изменяются при смене направления тока. В итоге среднее значение поверхностного сопротивления рассчитывается по формуле:

$$
\mathrm{R}_{\square}=\frac{\pi}{2 \ln 2}\left[\frac{V_{23}+V_{\text {of }}+V_{\text {TEMF }}}{I_{14}}+\frac{-V_{23}+V_{\text {of }}+V_{\text {TEMF }}}{-I_{14}}\right]=\frac{\pi}{\ln 2} \frac{V_{23}}{I_{14}} \text {. (6) }
$$

Более подробно данную методику демонстрирует рис. 6. Поверхностное сопротивление эпитаксиальных, легированных, диффузионных или осажденных пленок позволяет определить качество технологического процесса. Степень однородности характеристик слоя на поверхности подложки показывает расхождение параметров конечных кристаллов в разных местах на пластине. Именно поэтому

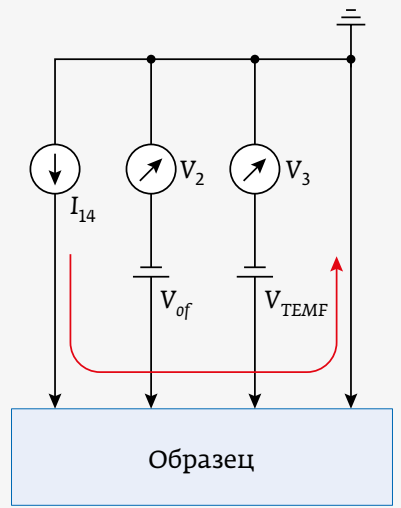

a)

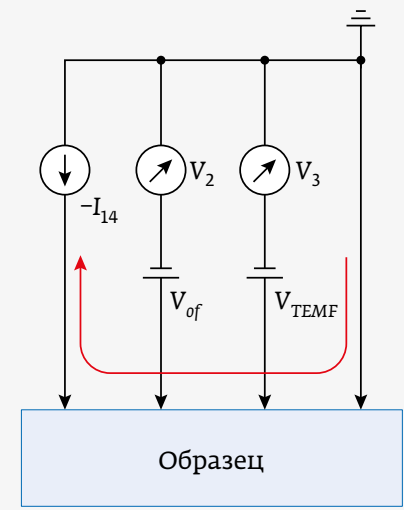

б)
Рис. 6. Исключение термо-ЭДс и смещения измерителя напряжения при смене направления тока

очень важно иметь возможность строить карты распределения поверхностного сопротивления по всей поверхности образца. Традиционный четырехзондовый метод и метод самокомпенсации геометрических эффектов успешно справляются с этой задачей и являются наиболее распространенными способами, которые реализованы на сегодняшний день во множестве различных установок разных производителей. Ниже мы рассмотрим основные типы установок и важные особенности, которые позволяют провести корректные и точные измерения электрофизических параметров образцов.

\section{ИЗМЕРИТЕЛЬНЫЕ КОМПЛЕКСЫ}

В советское время наиболее популярным был прибор иУс-3 (рис. 7а). Он включает в себя четырехзондовую голову, способную плавно опускаться за счет своей тяжести. Встроенный источник-измеритель проводит измерение поверхностного сопротивления, которое может
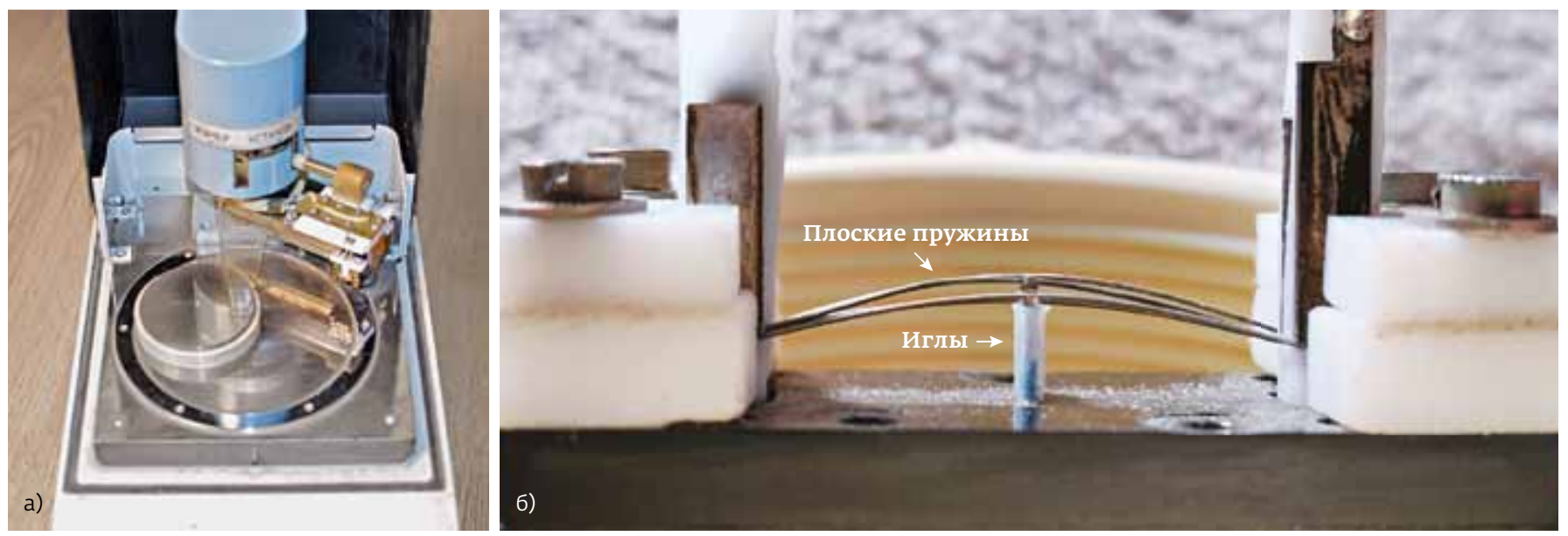

Рис. 7. Установка ИУС-3: а - внешний вид; 6 - конструкция измерительной головы 

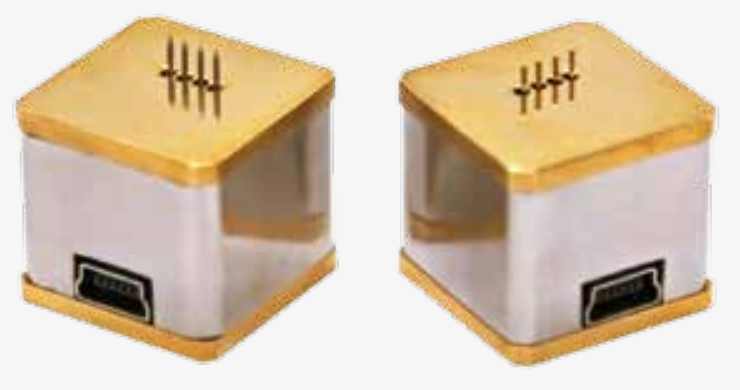

Рис. 8. Измерительные головы производства ООО «Остек-Электро"

быть использовано для расчета удельного сопротивления в случае однородного образца. Основным недостатком такой системы является измерительная голова, которая в силу отсутствия на тот момент технологии подпружиненных пробников была реализована на плоских пружинах (рис. 7б). Из-за этого головы быстро приходили в негодность и на данный момент такие системы требуют замены в связи с отсутствием производства расходных элементов.

Однако современные технологии позволили создать более конкурентное решение, способное выполнять порядка миллиона контактирований с воспроизводимостью 20 мкм. На сегодняшний день ООО «Остек-Электро» освоило производство измерительныхголов для измерения поверхностного и удельного сопротивлений четырехзондовым методом (рис. 8). Благодаря собственному производству такие параметры головы, как усилие прижатия, расстояние между иглами и радиус закругления игл, могут быть подобраны под конкретные исследуемые материалы. В качестве игл используются надежные подпружиненные пробники немецкой компании INGUN. Tехнология монтажа пробников предусматривает их установку в посадочную гильзу, исключающую люфт и латеральное перемещение иглы во время контакта. Такие измерительные головы также успешно применяются в автоматических установках.

В качестве преемника установки ИУс-3 ООО «Остек-Электро» успешно поставляет собственную разработку иус-7 (рис. 9). Установка содержит высокоточный источник-измеритель Keithley серии 2400 с базовой погрешностью 0,012\% (внесен в Госреестр (И), ручное контактирующее устройство с возможностью плавной регулировки усилия прижатия и быстрой замены измерительной головы,

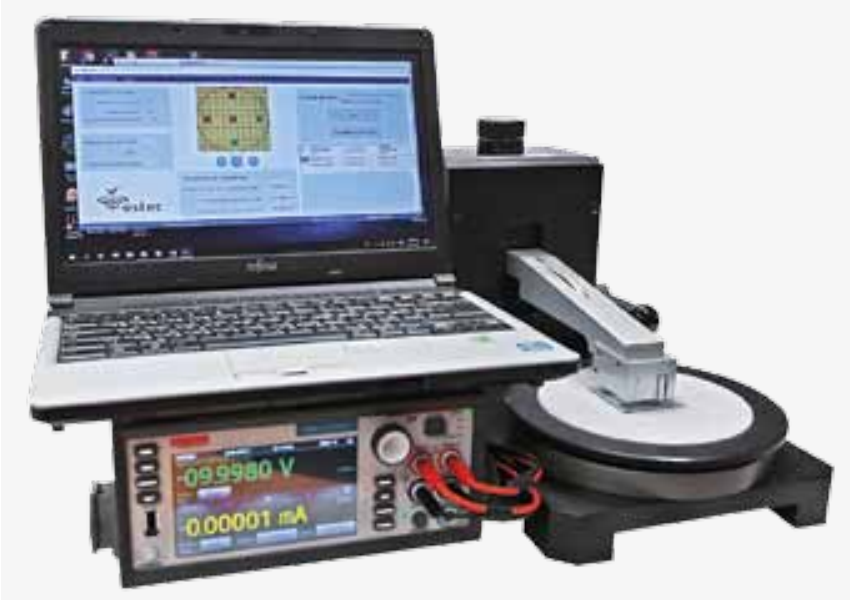

Рис. 9. Внешний вид установки ИУС-7

персональный компьютер с программным обеспечением «Кристалл» (рис. 10). Программное обеспечение позволяет учесть конкретные размеры образца и рассчитать необходимые поправочные коэффициенты согласно стандарту ASTM F84-99. Функция автоматического протоколирования сохраняет измеренные данные с привязкой к месту измерения на образце и выводит всю необходимую статистику в отчете. Автоматическая подстройка тока исключает инжекцию неосновных носителей заряда в образец и нагрев образца во время измерения. При необходимости установка может быть оснащена термостабилизированным столом. Данный программно-аппаратный комплекс полностью закрывает вопрос проведения измерений в ручном режиме.

Как уже было сказано ранее, для оценки качества технологического процесса необходимо получить распределение удельного и поверхностного сопротивлений на

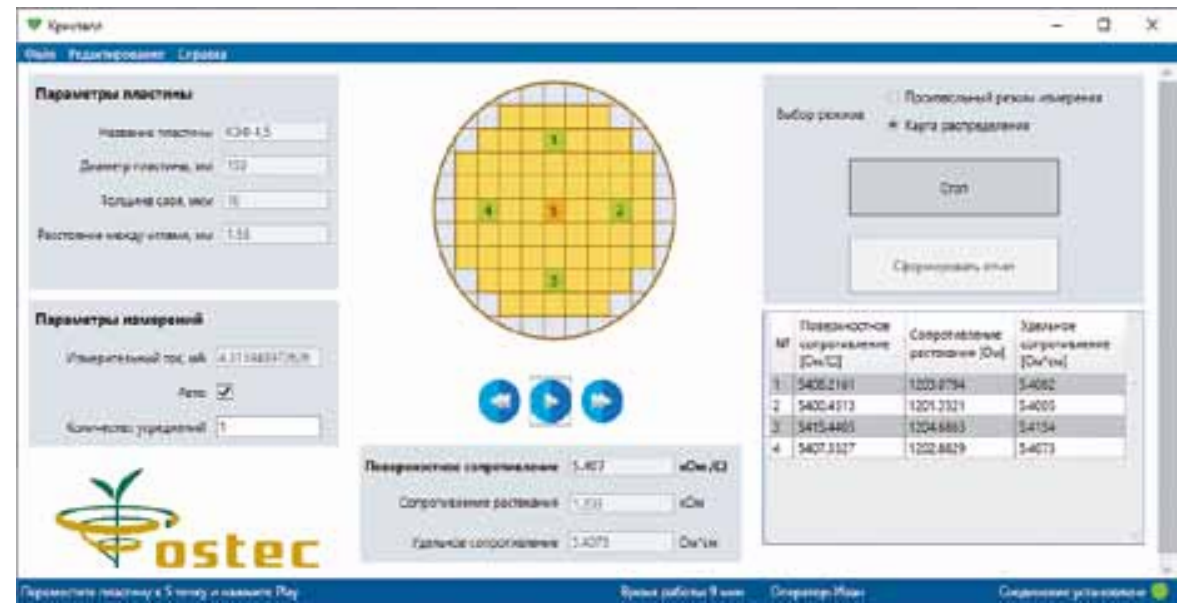

Рис. 10. Графический интерфейс пользователя программного обеспечения "Кристалл" 


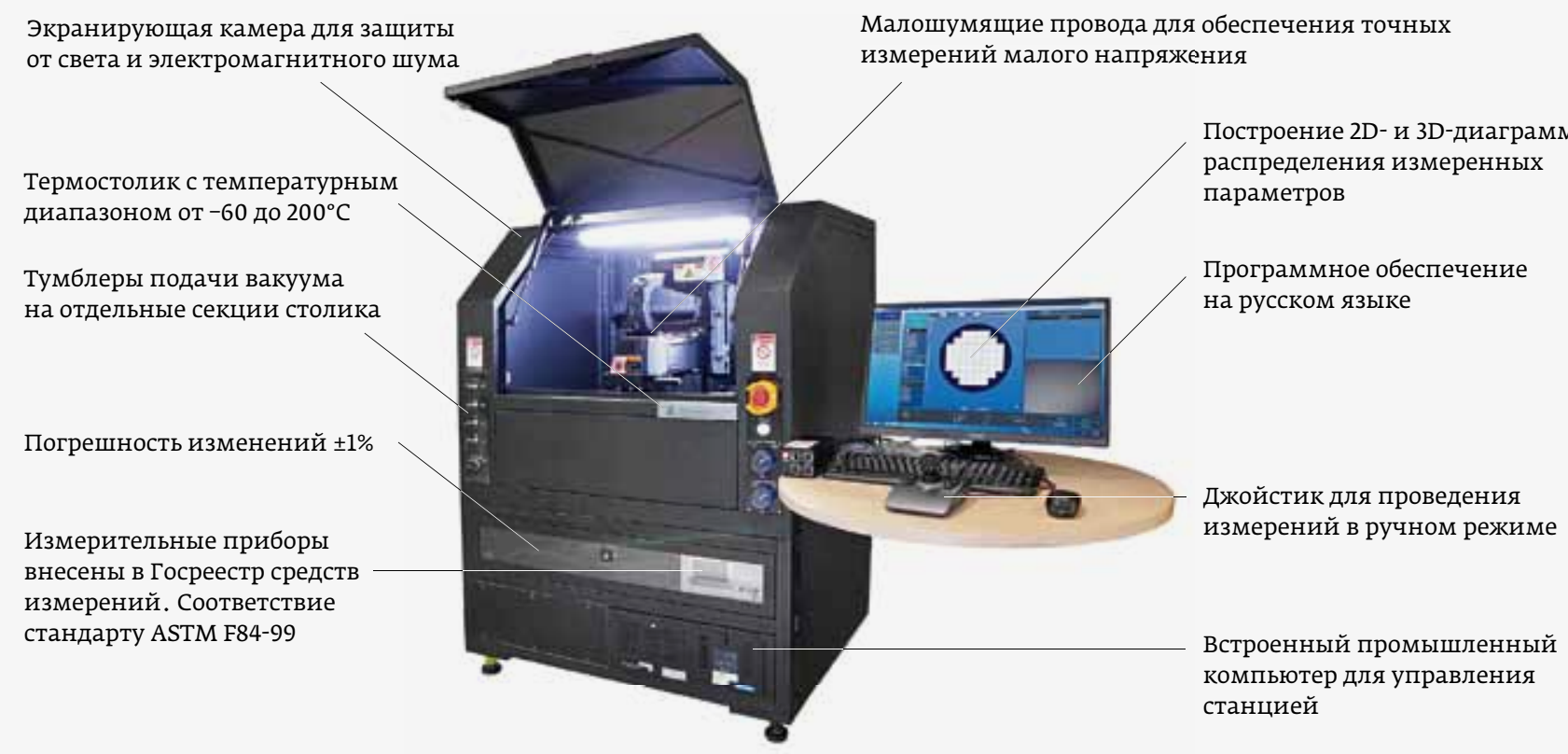

Рис. 11. Автоматическая установка для измерения удельного и поверхностного сопротивлений SF-P1500

всей поверхности образца. В этом случае требуются автоматические установки, способные перемещать измерительную голову либо стол и проводить измерения по заранее созданному рецепту без участия оператора. Установкa SF-P1500 (рис. 11), разработанная ООО "Остек-Электро» совместно с тайванской компанией Pomme Technologies, способна проводить измерения распределения поверхностного и удельного сопротивлений в том числе методом самокомпенсации геометрических эффектов.

Образец располагается на столе с вакуумным прижимом. Перемещение измерительной головы по поверхности образца осуществляется с помощью прецизионных приводов. Важным здесь является надежный и воспроизводимый контакт с образцом, так как это напрямую связано с точностью измерений: прохождение игл сквозь исследуемый слой вследствие чрезмерного прижатия может привести к получению ошибочных измерений и повреждению самого образца. Кроме высокоточного перемещения, требуется также изолировать образец от внешних вибраций. Для этих целей используется специальное виброизоляционное основание на воздушных подушках.

Фотопроводимость и фотоэффект могут значительно влиять на результаты измерений при работе с высокорезистивными полупроводниковыми слоями. Чтобы исключить влияние этих эффектов, образец располагается внутри камеры, которая ослабляет воздействие света и внешних электромагнитных полей. Кроме того, все измерительные кабели экранированы и расположены отдельно от кабелей питания.

В программном обеспечении с русскоязычным интерфейсом оператор создает тестовый рецепт, в котором указывает необходимое количество точек на образце, величину измерительного тока, температуру стола ит.д. Затем установка в автоматическом режиме производит измерения согласно рецепту и рассчитывает удельное и поверхностное сопротивления в соответствии со стандартами ASTM и SEMI. Полученные данные подвергаются статистической обработке. Результат измерений представляется в виде таблицы или как 2D / 3D-диаграмма (рис. 12).

Помимо измерения удельного и поверхностного сопротивлений, установка SF-P1500 имеет опцию встроенного термостатирующего стола. Температурный диапазон может быть подобран в зависимости от решаемой задачи. С помощью этой опции удается замерить не только удельное и поверхностное сопротивления, но и температурный коэффициент сопротивления (ТКС), который позволяет получить дополнительную информацию об образце при различных температурах.

С точки зрения метрологии как ручные, так и полуавтоматические установки являются комплексными средствами измерений, которые состоят из источника-измерителя, соединительных кабелей и измерительной головы. Поэтому даже если источник-измеритель внесен в Госреестр СИ, необходимо непосредственно убедиться, что результаты измерений не искажаются наводками в кабелях или неправильным расположением игл на образце. Это возможно с помощью использования стандартных образцов. Желательно, чтобы стандартный образец как можно больше соответствовал по характеристикам реальным образцам, на которых проводятся измерения. По результатам аттестации на каждый образец выдается сертификат, 
Оператор: Иван

Дата: $\quad 27.04 .2020$

Время: $\quad 14: 44$

\begin{tabular}{|l|l|}
\hline \multicolumn{2}{|l|}{ Параметры пластины } \\
\hline Тип пластины & Круглая \\
\hline Диаметр пластины, мм & 50 \\
\hline Толщина пластины, мкм & 100 \\
\hline Расстояние между иглами, мм & 1,5875 \\
\hline Измерительный ток, мА & 1 \\
\hline
\end{tabular}

\begin{tabular}{|c|c|}
\hline \multicolumn{2}{|c|}{$\begin{array}{l}\text { Статистика распределения поверхностного } \\
\text { сопротивления по пластине }\end{array}$} \\
\hline Среднее значение, Ом/口 & 47,3431 \\
\hline Стандартная ошибка, Ом/口 & 0,295754 \\
\hline Медиана, Ом/ロ & 47,2103 \\
\hline Стандартное отклонение, Ом/ロ & 0,661325 \\
\hline 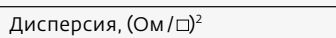 & 0,4373513039 \\
\hline
\end{tabular}

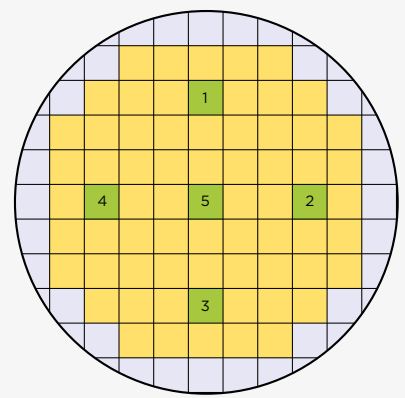

\begin{tabular}{|l|l|l|l|}
\hline \multicolumn{2}{|l|}{ Измеренные параметры } \\
\hline $\begin{array}{l}\text { № } \\
\text { точки }\end{array}$ & $\begin{array}{l}\text { Поверхностное } \\
\text { сопротивление, Ом/口 }\end{array}$ & $\begin{array}{l}\text { Сопротивление } \\
\text { растекания, Ом }\end{array}$ & $\begin{array}{l}\text { Удельное } \\
\text { сопротивление, Ом·см }\end{array}$ \\
\hline 1 & 47,1257 & 10,5481 & 0,4713 \\
\hline 2 & 48,2961 & 10,8101 & 0,483 \\
\hline 3 & 47,5871 & 10,6514 & 0,4759 \\
\hline 4 & 47,2103 & 10,5671 & 0,4721 \\
\hline 5 & 46,4964 & 10,4073 & 0,465 \\
\hline
\end{tabular}

Рис. 12. Пример отчета о результатах измерений с итоговой статистикой дополнительный источник погрешности, связанный с температурой самого образца: так как образец имеет толщину, то верхний его слой всегда будет иметь отличную от стола температуру. В этом случае используется специальный резистор, изготовленный на теплопроводящей подложке, имитирующей подложку исследуемого образца (рис. 13). Его аттестация проходит в камере тепла-холода, где исключается наличие неравномерного нагрева. После этого терморезистор располагается непосредственно на столе и производятся измерения ТКС с помощью установки. Сопоставив полученные результаты измерения ТКС в камере тепла-холода и на термостатирующем столе, можно оценить влияние градиента температуры по толщине образца и точности установки температуры стола на результаты измерения.

$$
\therefore *
$$

Несмотря на кажущуюся простоту четырехзондового метода измерения удельного и поверхностного сопротивлений возникает множество трудностей при его реализации на практике. Мы рассмотрели основные моменты, на которые стоит обратить внимание при выборе оборудования, а также способы оценки погрешности результатов измерения. Однако при работе с определенными материалами могут возникать дополнительные нежелательные затруднения. В этом случае перед выбором конкретной установки рекомендуется провести реальные измерения, на основании которых подобрать обеспечивающую достоверные измерения конфигурацию. Такой подход позволит сэкономить и деньги, и время.

ООО «Остек-Электро» обладает многолетним опытом в поставке программно-аппаратных комплексов для измерения удельного и поверхностного сопротивлений: начиная от собственной разработки и сборки измерительных голов и заканчивая написанием программного обеспечения для автоматических установок. Компания имеет необходимое оборудование в своем демонстрационном зале, что позволяет увидеть и провести замеры на реальных образцах. Высококвалифицированные инженеры помогут подобрать необходимые опции или разработать необходимые узлы непосредственно под конкретные требования. Именно такой подход позволит безошибочно найти лучшее решение, особенно для нестандартных задач.
Рис. 13. Терморезистор для оценки погрешности измерения ТКС 


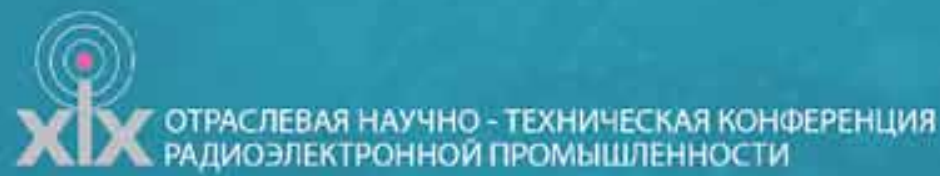

РАДИОЭЛЕКТРОННОИ ПРОМЫШШЕННОСТИ

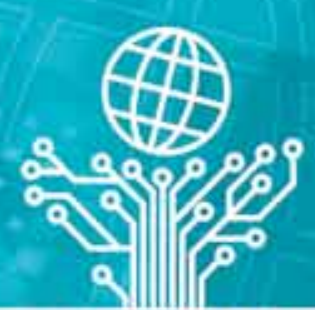

МЕЖДУНАРОДНЫЙ ФОРУМ МИКРОЭЛЕКТРОНИНА-202О

28 сентября - 3 октября

Респубпика Крым, г. Япта

\section{XIX Отраслевая научно-техническая конференция радиоэлектронной промышленности}

ключевое событие года в области микроэлектронных технологий

Международный Форум «Микроэлектроника 2020»

V Научная конференция «ӘКБ и микрояпектронные модупи»

V Деловая программа V Демо-зона

V INRADEL/Фестиваль инноваций $\quad$ V Школа молодых ученых

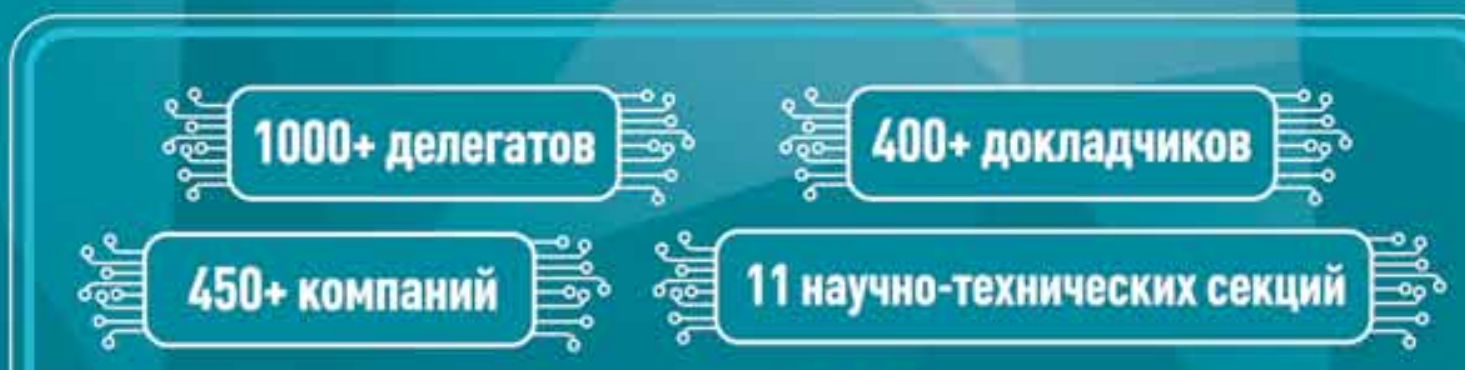

5 лет успешной работы

ОРГАНИЗАТОРЫ

МИНПРООМТОРГ
8 НИИММЭ

CTPATETИЧЕСКИЙ ПАРTHËP Партнер INRADEL/ФестивапЯ инНоваций

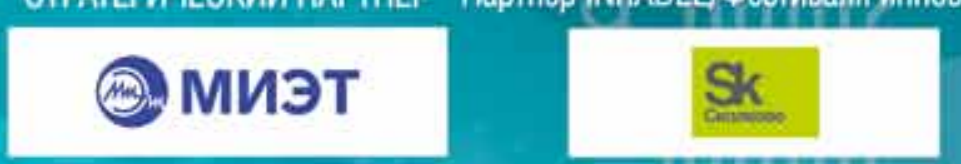

\section{1)}

ПPORPECC

IIPOTPECC
ПРИ ПОДДЕРЖКЕ

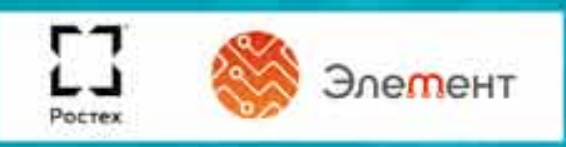

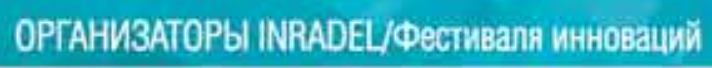

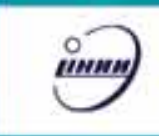

\section{INR/DEL}

ГЕНЕРАЛЬНЫЙ ИНФОРМАЦИОННЫЙ ПАРТНЁР

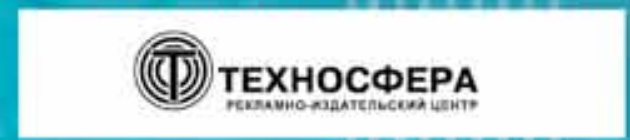

Teл: + +7 (495) 641-57-17 • E-mail: inio@microelectronica.pro

Подробная информация об Отраслевой конференции и форуме и регистрация: drepconffru, microelectronica.pro 